\title{
Gender Differences in Multiple Endocrine Neoplasia Type 1: Implications for Screening?
}

\author{
Jerena Manoharan ${ }^{a}$ Carmen Bollmann ${ }^{a}$ Peter Herbert Kann ${ }^{b}$ Pietro Di Fazio ${ }^{a}$ \\ Detlef K. Bartsch ${ }^{a}$ Max B. Albers ${ }^{a}$ \\ a Department of Visceral, Thoracic and Vascular Surgery, Philipps University Marburg, Marburg, Germany; \\ ${ }^{b}$ Division of Endocrinology, Philipps University Marburg, Marburg, Germany
}

\section{Keywords}

Multiple endocrine neoplasia type $1 \cdot$ Screening $\cdot$ Gender

\begin{abstract}
Background: Some gender-related differences have been reported in multiple endocrine neoplasia type 1 (MEN1), although not all reports are conclusive. This systematic review with analysis of the own MEN1 cohort evaluates gender differences and potential consequences for screening. Methods: A systematic review of the literature between 1990 and 2019 with the search terms "MEN1" or "multiple endocrine neoplasia type 1" and "gender" or "sex" was performed. In addition, the prospectively collected data of a genetically confirmed MEN1 cohort of the Philipps University Marburg were retrospectively analyzed. Results: Review of the literature identified five retrospective case series with original data of 1,057 MEN1 patients. One series suggested a higher frequency of pancreatic neuroendocrine neoplasms (NEN), especially gastrinomas, in men (61 vs. $54 \%$ ) and a higher frequency of pituitary tumors in women (47 vs. 30\%), but others did not. Only thymic NEN occurred predominantly in men throughout all studies. Women with MEN1 were found to have an increased risk of breast cancer. In the own series consisting of 116 MEN1 patients (male = 58 , female $=58$ ), thymic lesions were also more frequently detected in male patients (male $=5$, female $=1$ ). No gender difference was found with regard to the other manifestations. Conclusion: Regarding the typical MEN1 tumor man-
\end{abstract}

ifestations, gender-adapted diagnostic and therapeutic approaches cannot be recommended. Female MEN1 patients should be encouraged to participate in breast cancer screening programs.

() 2020 S. Karger AG, Basel

\section{Introduction}

Multiple endocrine neoplasia type 1 (MEN1) is a rare autosomal dominant inherited tumor syndrome caused by germline mutations of the MENIN gene on chromosome $11 \mathrm{q} 13$ [1]. The penetrance of the disease approximates $100 \%$ by the age of 50 years [2,3]. Manifestations classically include primary hyperparathyroidism (pHPT), duodenopancreatic neuroendocrineneoplasias (dpNEN), and pituitary adenomas. Other detectable lesions are bronchial and thymic NEN, as well as adrenal lesions. Recently, an increased risk of breast cancer has been described for women with MEN1 [4]. From genetic considerations and observational studies, the distribution of typical MEN1 lesions has been assumed to be almost equal in men and women [5].

Current practice guidelines by experts $[5,6]$ and ENETS centers of excellence $[2,7]$ recommend regular screening in MEN1 patients beginning by the age of 6-16 years. The goal of screening is the early detection of any organ manifestation to prevent malignant spread and preserve the quality of life. Screening should include lab- 
Fig. 1. Results of the systematic literature

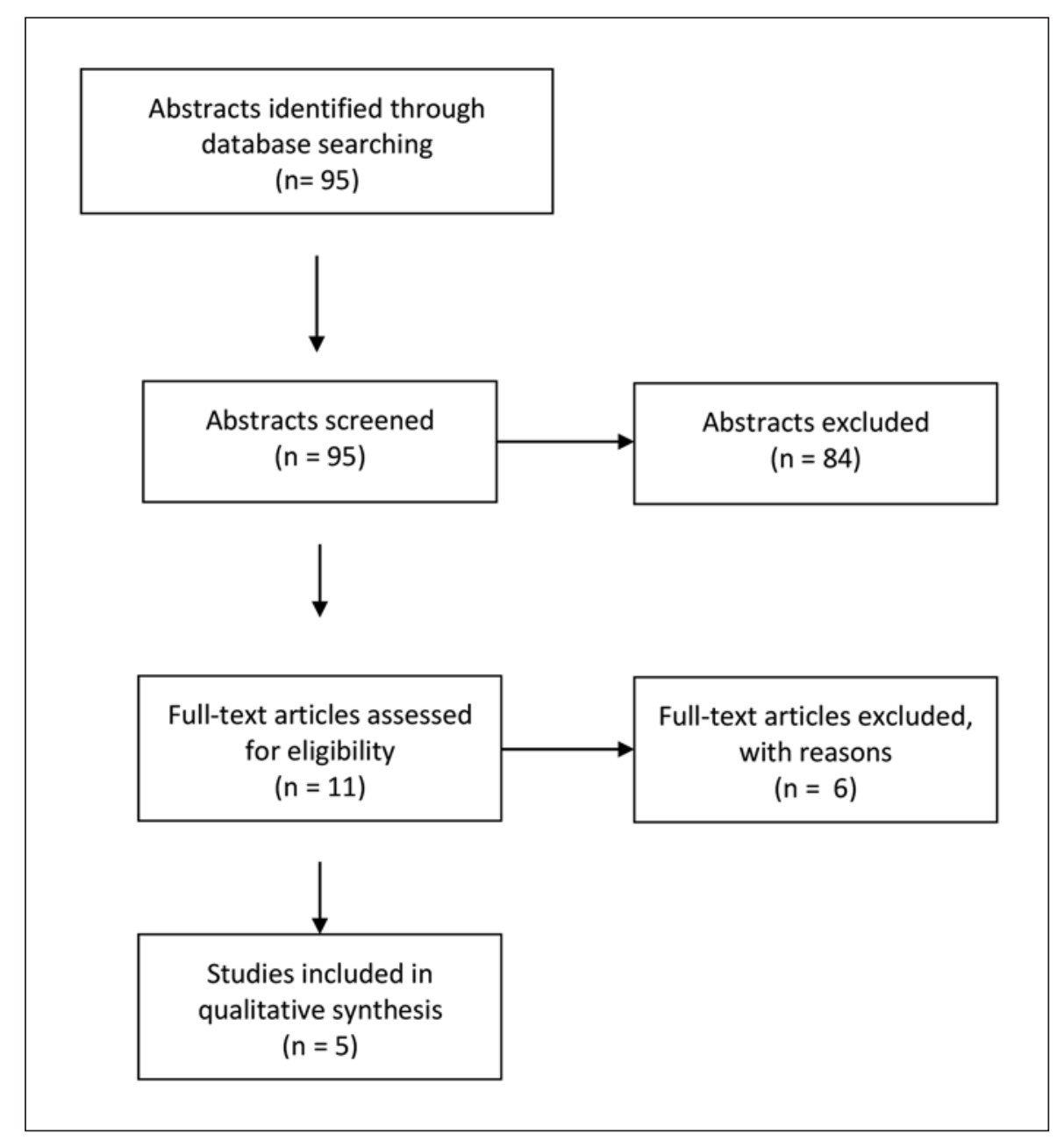
search.

oratory tests for endocrine dysfunction and imaging procedures of the target organs with potential neoplastic manifestations of the syndrome. In addition, annual screening of asymptomatic MEN1 patients seems to be of benefit, but it has to be noted that screening implicates costs and psychological burden [7]. Current guidelines do not refer to gender-specific screening intervals or techniques $[2,5]$. However, some recent studies have shown gender-dependent prevalence of some MEN1-asscociated organ manifestations $[3,8,9]$.

The aim of this study was to analyze gender differences in organ manifestations in MEN1 patients and whether those, if present, should have consequences for screening programs.

\section{Methods}

\section{Systematic Review}

Search Strategy

The Medline database was searched for the terms "MEN1" or "multiple endocrine neoplasia type 1" and "gender" or "sex" to identify studies analyzing gender differences in MEN1 published between 1990 and today. The initial search was performed in October 2019 and was updated in November 2019. Initially, titles and abstracts of the articles were screened. All articles reporting original data of $\geq 50 \mathrm{MEN} 1$ patients were included. Articles published in other languages than English or German were excluded from the literature search. Furthermore, duplicates were excluded. In case of multiple publications of data from the same cohort, only the most recent or the one with the most detailed information on gender differences was analyzed.

Three authors (J.M., D.K.B., M.B.A.) of this study independently assessed the search results for inclusion. Data of included studies was extracted regarding study design, study population, patient characteristics, organ manifestations, and patient followup. The reference lists of all relevant articles were reviewed as well to identify further suitable publications.

\section{Marburg MEN1 Database}

Data of genetically confirmed MEN1 patients who were treated at the Department of Visceral, Thoracic and Vascular Surgery at the Philipps University Marburg, Germany, have been collected in a prospective database since 1997 after approval of the local ethical committee, and were retrospectively analyzed with special regards to gender-dependent screening results and tumor manifestations. Data of some patients have been previously reported [10,11]. The diagnostic criteria for MEN1 and annual screening followed a standardized protocol as previously published $[2,7]$. Regular 
screening included measuring of plasma hormone levels [2] and imaging. Abdominal imaging comprised annual magnetic resonance imaging (MRI) and endoscopic ultrasonography. In addition, magnetic resonance imaging of the pituitary and thoracic computed tomography (CT) were performed at 1- or 3-year intervals according to previously identified lesions. Somatostatin receptor scintigraphy was performed for functional imaging until 2012. In 2013 and 2014, Ga68-DOTATOC-PET/CT was performed on all patients during regular screening [12]. Since 2015, Ga68-DOTATOC-PET/CT has only been performed in patients with suspected metastatic disease.

Statistical Analysis

Data were analyzed using the SPSS software (version 26; SPSS, Inc.). Nonparametric data are presented as median and range. $p$ values $<0.050$ were considered statistically significant. Fisher's exact test or the $\chi^{2}$ test were used for crude analysis of dichotomous data.

\section{Results}

\section{Review of the Literature}

PubMed search of the terms "MEN1" or "multiple endocrine neoplasia type 1" and "gender" or "sex" could identify 95 abstracts, all published after 1990. After screening of all 95 titles and abstracts, a total of 11 full texts were assessed for eligibility. Six studies were excluded because of repetitive publication of data from the same cohort. A total of 5 studies fulfilled the inclusion criteria $[4,8,10,11,13]$ (Fig. 1). Two studies reported data from the Marburg cohort, which were also analyzed in the present original data. Screening of the reference lists could not reveal further eligible studies.

All 5 eligible studies were retrospective case series. Original data on gender differences of 1,057 patients with MEN1 were identified, including 503 men and 669 women, not including patients from the presently reported Marburg cohort (Table 1).

Only one study by Goudet et al. [8] comprehensively assessed gender differences regarding all manifestations of 734 patients (310 men, 424 women) with MEN1 from various centers in France and Belgium. The authors found a higher frequency of the Zollinger-Ellison syndrome (ZES) in men $(36.5 \%)$ than in women $(24.3 \%, p<0.001)$ and a higher frequency of pituitary tumors in women $(46.5 \%)$ than in men $(30.3 \%, p<0.001)$. Thymic tumors occurred exclusively in $19(6.1 \%)$ male patients $(p<0.001)$ (Table 1$)$.

de Laat et al. [13] provided detailed information of 27 patients with thymic and pulmonal NEN among 135 men and 188 women with MEN1 from the Dutch MEN1 study group. Whereas the incidence of pulmonal NEN did not differ between gender, thymic NEN were also significantly more frequent, but not exclusive, in men (91 vs. 9\%).

Furthermore, within a multicentric MEN1 cohort from the Netherlands, France, and Belgium, the risk of breast cancer was significantly higher than in the reference population [4].

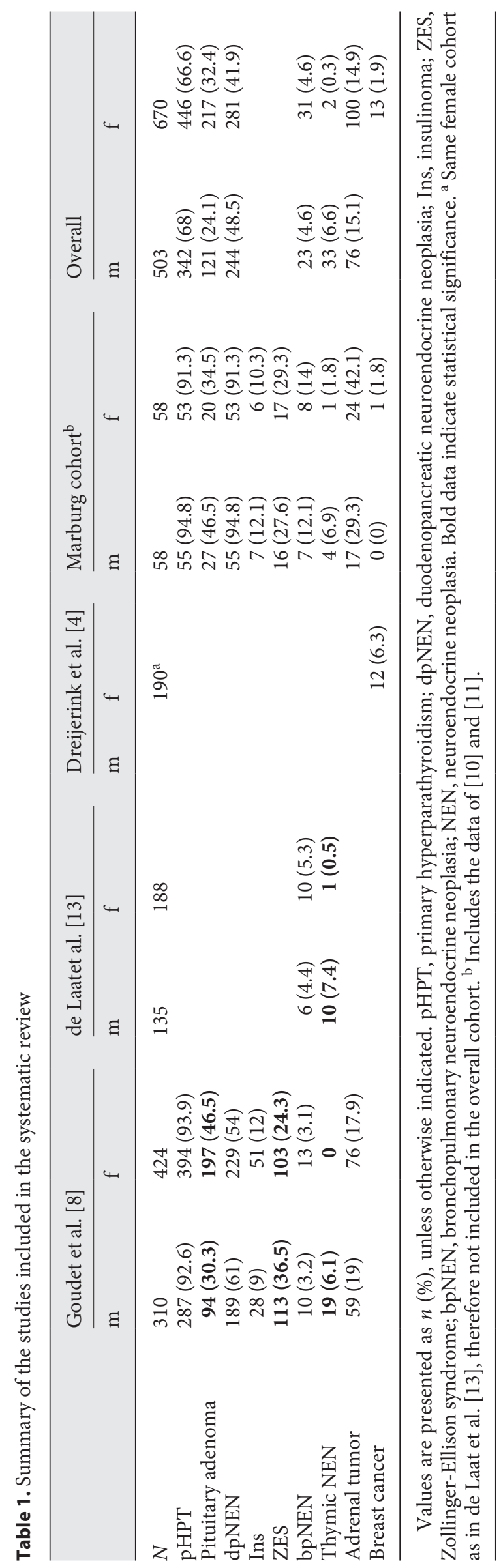


Table 2. Gender-dependent organ manifestations in the Marburg MEN1 cohort

\begin{tabular}{lcccc}
\hline & All $(n=116)$ & Male $(n=58)$ & Female $(n=58)$ & $p$ value \\
\hline pHPT & $108(93.1)$ & $55(94.8)$ & $53(91.3)$ & 0.464 \\
Pituitary adenoma & $47(40.5)$ & $27(46.5)$ & $20(34.5)$ & 0.211 \\
dpNEN & $108(93.1)$ & $55(94.8)$ & $53(91.3)$ & 0.464 \\
$\quad$ ZES & $33(28.7)$ & $16(27.6)$ & $17(29.3)$ & 0.837 \\
Insulinoma & $13(11.2)$ & $7(12.1)$ & $6(10.3)$ & 0.769 \\
$\quad$ NF-pNEN & $79(68.1)$ & $42(72.4)$ & $37(63.8)$ & 0.319 \\
Bronchial NEN & $15(12.9)$ & $7(12.1)$ & $8(14)$ & 0.754 \\
Thymic NEN & $5(4.3)$ & $4(6.9)$ & $1(1.8)$ & 0.176 \\
Adrenal lesions & $41(35.3)$ & $17(29.3)$ & $24(42.1)$ & 0.152 \\
Breast cancer & $1(0.9)$ & $0(0)$ & $1(1.8)$ & 0.311 \\
\hline
\end{tabular}

Values are presented as $n(\%)$. pHPT, primary hyperparathyroidism; dpNEN, duodenopancreatic neuroendocrine neoplasia; NEN, neuroendocrine neoplasia; NF-pNEN, non-functioning pancreatic neuroendocrine neoplasia; ZES, Zollinger-Ellison syndrome.

Lopéz et al. [11] described a significantly smaller body height in female MEN1 patients as compared to their first degree-relatives and the German female population, which was not as evident in male MEN1 patients.

Finally, Bartsch et al. [10] provided detailed information on a cohort of patients with MEN1 and found a higher prevalence of dpNEN in male patients compared to women (100 vs. $88 \%, p=0.042)$. No significant gender difference was found for thymic, bronchial, or other malignant neuroendocrine tumor (NET).

None of the reviewed studies reported gender-specific differences in the incidence of hyperparathyroidism, adrenal tumors, or bronchopulmonary NET. The systematic review of gender-associated organ manifestations of MEN1 are summarized in Table 1.

\section{Marburg Cohort}

In total, 116 patients with MEN1 syndrome have been treated at our institution. Men $(n=58,50 \%)$ and women $(n=58,50 \%)$ were equally distributed. Nine $(7.8 \%)$ out of the 30 (25.9\%) MEN1 patients who did not participate in annual screening have been lost to follow-up. Eleven $(9.5 \%)$ out of 116 MEN1 patients died because of a MEN1-related tumor after a median follow-up of 138 (range 12-420) months.

Of the 116 MEN1 patients, 107 (93\%) were diagnosed with $\mathrm{pHPT}$ with no gender-specific prevalence $(p=$ 0.448). Pituitary lesions were found in 47 (40.9\%) MEN1 patients, including 27 male and 20 female patients with no gender-specific difference $(p=0.211)$. In total, 108 (93\%) patients developed dpNEN, of whom 55 were male and 53 were female MEN1 patients with no genderspecific difference $(p=0.448)$. Out of the 108 MEN1 patients with dpNEN, 33 (28.7\%) developed Zollinger-Ellison syndrome $($ male $=16$, female $=17) ; 13(11.2)$ developed insulinoma $($ male $=7$, female $=6)$; and $79(68.1 \%)$
MEN1 patients developed a nonfunctioning pNEN. The different dpNEN types did not show gender-specific differences (Table 2).

Bronchial NEN were diagnosed in 15 (13\%) MEN1 patients ( 7 males and 8 females, $p=0.754)$. NETs of the thymus were detected in $5 \mathrm{MEN} 1$ patients. One of these 5 patients was female $(p=0.176)$. Adrenal lesions were discovered in 41 (35.3\%) MEN1 patients, including 17 male and 24 female patients $(p=0.15)$.

Breast cancer was detected in only $1(0.9 \%)$ female patient. None of the male MEN1 patients developed breast cancer. The results of organ manifestations in the Marburg cohort are summarized in Table 2.

\section{Discussion}

Regular screening at an expert center is recommended for all patients with MEN1, as the penetrance of the disease is nearly $100 \%$ by the age of 50 years. Early detection of manifestations can prevent metastasis of malignant tumors leading to premature death and help patients maintain a good quality of life $[5,7,14]$. Screening recommendations of current guidelines do not differentiate between male and female patients [5]. The present study analyzed gender differences of MEN1 organ manifestations in 1,057 MEN1 patients from the literature and 116 MEN1 patients from the Marburg cohort. There are only very few retrospective studies somehow focusing on gender differences in MEN1. Some of these retrospective registry-based studies cover very long time periods and show a large data heterogeneity as well as certain methodical problems. Thus, the data analyzed in the presented systematic review have to be interpreted with caution.

Given the autosomal dominant trait of MEN1, the gender distribution should be equally balanced. The Marburg 
cohort comprised an equal number of men and women, but the systematic review revealed a slight predominance of women of about $60 \%$ [8, 15-17]. However, since none of these retrospective studies are population based, this inequality is most likely caused by selection bias.

In the present systematic review and in the Marburg cohort, no gender differences have been observed in the prevalence of $\mathrm{pHPT}$, adrenal lesions, or bronchopulmonary NEN. For the prevalence of dpNEN, pituitary adenomas, and thymic NEN, however, gender differences have been described by some authors $[8,10,13]$. Another recent finding highlighted that the prevalence of breast cancer might be elevated in female MEN1 patients [4]. These suggested, but not confirmed, gender differences as well as their potential implications on screening recommendations are discussed below.

\section{Duodenopancreatic Neuroendocrine Neoplasms}

In a large cohort of MEN1 patients from the Groupe d'Etude des Tumeurs Endocrines (GTE), and within a unicentric German patient cohort, the incidence of duodenopancreatic NEN (dpNEN) was significantly higher in men than in women $[8,10]$. In the GTE cohort, the higher frequency of dpNEN in men was mostly due to a higher prevalence of ZES in male patients (113 of 310 men, vs. 103 of 424 women). As the authors critically discussed, data from their cohort dates back as far as to 1951 when the MEN1 syndrome was hardly understood, diagnostic options were limited, and the diagnosis was often only made in patients with advanced and symptomatic disease. In the earlier Marburg cohort with 76 patients, the prevalence of dpNEN was $100 \%$ in men and $88 \%$ in women $(p=0.04)$ [10]. In the presented updated Marburg cohort, however, the prevalence of dpNEN is around $93 \%$ without gender differences, neither overall nor functioning nor nonfunctioning. Approximately 20 years ago, the estimated incidence of dpNEN in MEN1 patients was below 50\% [17]. Based on improved imaging techniques and the conduction of regular screening at expert centers, it becomes now more evident that the incidence of dpNEN is above $90 \%$ until the age of 70 years [18]. Furthermore, it is now well understood that gastrinomas in patients with MEN1ZES arise almost exclusively in the duodenum and very rarely in the pancreas [19]. The earlier reported incidences of dpNEN must therefore be interpreted with caution. It has also been reported that the prognosis of dpNEN metastatic to the liver might be worse in men than in women, which will not have any influence on regular screening [20]. Given the fact that dpNEN develop frequently in both sexes, and metastatic pNEN disease is the most common disease-related cause of death, screening for the presence of all types of dpNEN should be performed thoroughly in both sexes with adequate laboratory tests and at least one imaging modality in annual intervals.

\section{Pituitary Adenomas}

Pituitary adenomas have been described to be more frequent in women than men ( 46.5 vs. $30.3 \%$ ) in one large French-Belgian series [8]. As for ZES, this might be related to the more likely diagnosed endocrine dysfunction in women who may have symptoms of erratic menstruation and infertility [21], as the majority of pituitary tumors in women from this cohort were prolactinomas $(54,8 \%)$. This finding could not be confirmed in the other reports included in the systematic review nor in the updated Marburg cohort. Here, pituitary tumors occurred even more often in men, although without statistical significance. Thus, a gender-specific prevalence of pituitary adenomas remains controversial. However, since the prevalence is $>40 \%$ in both sexes, screening for pituitary adenomas should be performed on a regular basis.

\section{Thymic NEN}

Thymic NEN are considered the most aggressive manifestation of MEN1, which therefore represent the second most common disease-related cause of death in MEN1 in spite of their rarity of approximately $5 \%[5,8,10,11]$. As the present systematic review, several other reports have shown consistently that thymic NEN affect much more often men than women $[9,22,23]$, although the gender ratio of 20:1 is only an estimate due to the small number of cases. Despite thymic NEN are very rare, especially in MEN1 women, biannual imaging of the chest is recommended for both sexes to detect bronchopulmonary NEN, which develop equally frequent in men and women, namely in up to $30 \%$ of the patients [10]. Thus, regular chest imaging is indicated in both sexes.

\section{Breast Cancer}

Following up on a finding in an animal model of MEN1, the Dutch MEN1 study group [4] reported an increased risk for (mostly luminal) breast cancer in women with the MEN1 syndrome. In the Dutch cohort, 12 out of 190 women $(6.3 \%)$ were affected. The relative risk of breast cancer in this series was 2.83 when compared to the general Dutch female population. This finding could be supported within 675 female MEN1 patients from cohorts from the USA, France, and Tasmania, reporting a combined incidence ratio of 1.96 [4]. In the presented smaller Marburg cohort of 58 women, however, only 1 woman $(1.8 \%)$ developed breast cancer. This prevalence is not higher than that of the general German female population. Nevertheless, population-based, large-scale studies are needed to confirm the increased risk of breast cancer in female MEN1 patients. Women affected by the MEN1 syndrome should be encouraged to undergo breast cancer screening as offered by the respective governments and insurance companies. 


\section{Conclusion}

Regarding the typical MEN1 tumor manifestations, gender-differentiated diagnostic and therapeutic approaches cannot be recommended. Female MEN1 patients should be encouraged to participate in breast cancer screening programs.

\section{Acknowledgements}

We want to thank Caroline Lopez-Lopez, Peter Langer, Volker Fendrich, Jens Waldmann, Emily Slater, and Matthias Rothmund for the foundation and maintenance of the MEN1 database in Marburg.

\section{Statement of Ethics}

Data acquisition and analysis was approved by the Ethics Committee of the Philipps University of Marburg in compliance with the Helsinki Declaration. All patients gave written informed consent.

\section{Disclosure Statement}

The authors have nothing to disclose.

\section{Funding Sources}

This research did not receive any specific grant from any funding agency in the public, commercial, or not-for-profit sector.

\section{Author Contributions}

J.M.: Conception of the study, data acquisition, data analysis, data interpretation, drafting of the manuscript.

P.D.F.: Data acquisition, critical revision of the manuscript.

C.B.: Data acquisition, critical revision of the manuscript.

P.H.K.: Data acquisition, critical revision of the manuscript.

D.K.B.: Conception of the study, data acquisition, data analysis, data interpretation, critical revision of the manuscript.

M.B.A.: Conception of the study, data acquisition, data analysis, data interpretation, drafting of manuscript, critical revision of the manuscript.

All authors gave approval of the version to be published.

\section{References}

1 Chandrasekharappa SC, Guru SC, Manickam P, Olufemi SE, Collins FS, Emmert-Buck MR, et al. Positional cloning of the gene for multiple endocrine neoplasia-type 1. Science. 1997 Apr; 276(5311):404-7.

2 Manoharan J, Albers MB, Bartsch DK. The future: diagnostic and imaging advances in MEN1 therapeutic approaches and management strategies. Endocr Relat Cancer. 2017 Oct; 24(10):T209-25.

3 Machens A, Schaaf L, Karges W, Frank-Raue K, Bartsch DK, Rothmund M, et al. Age-related penetrance of endocrine tumours in multiple endocrine neoplasia type 1 (MEN1): a multicentre study of 258 gene carriers. Clin Endocrinol (Oxf). 2007 Oct;67(4):613-22.

4 Dreijerink KM, Goudet P, Burgess JR, Valk GD; International Breast Cancer in MEN1 Study Group. Breast-cancer predisposition in multiple endocrine neoplasia type 1. N Engl J Med. 2014 Aug;371(6):583-4.

5 Thakker RV, Newey PJ, Walls GV, Bilezikian J, Dralle H, Ebeling PR, et al.; Endocrine Society. Clinical practice guidelines for multiple endocrine neoplasia type 1 (MEN1). J Clin Endocrinol Metab. 2012 Sep;97(9):2990-3011.

6 Falconi M, Bartsch DK, Eriksson B, Klöppel G, Lopes JM, O'Connor JM, et al.; Barcelona Consensus Conference participants. ENETS Consensus Guidelines for the management of patients with digestive neuroendocrine neoplasms of the digestive system: well-differentiated pancreatic non-functioning tumors. Neuroendocrinology. 2012;95(2):120-34.

7 Manoharan J, Raue F, Lopez CL, Albers MB, Bollmann $\mathrm{C}$, Fendrich $\mathrm{V}$, et al. Is Routine Screening of Young Asymptomatic MEN1 Patients Necessary? World J Surg. 2017 Aug; 41(8):2026-32.

8 Goudet P, Bonithon-Kopp C, Murat A, Ruszniewski P, Niccoli P, Ménégaux F, et al. Gender-related differences in MEN1 lesion occurrence and diagnosis: a cohort study of 734 cases from the Groupe d'etude des Tumeurs Endocrines. Eur J Endocrinol. 2011 Jul;165(1): 97-105.

9 Goudet P, Murat A, Cardot-Bauters C, Emy P, Baudin E, du Boullay Choplin H, et al.; GTE network (Groupe des Tumeurs Endocrines). Thymic neuroendocrine tumors in multiple endocrine neoplasia type 1: a comparative study on 21 cases among a series of 761 MEN1 from the GTE (Groupe des Tumeurs Endocrines). World J Surg. 2009 Jun;33(6):1197207.

10 Bartsch DK, Slater EP, Albers M, Knoop R, Chaloupka B, Lopez CL, et al. Higher risk of aggressive pancreatic neuroendocrine tumors in MEN1 patients with MEN1 mutations affecting the CHES1 interacting MENIN domain. J Clin Endocrinol Metab. 2014 Nov;99(11):E238791.

11 López CL, Langer P, Waldmann J, Fendrich V, Sitter H, Nies C, et al. Shortness: an unknown phenotype of multiple endocrine neoplasia type 1. Eur J Endocrinol. 2013 Jul;169(1):133-7.

12 Albers MB, Librizzi D, Lopez CL, Manoharan J, Apitzsch JC, Slater EP, et al. Limited Value of Ga-68-DOTATOC-PET-CT in Routine Screening of Patients with Multiple Endocrine Neoplasia Type 1. World J Surg. 2017 Jun;41(6): 1521-7.

13 de Laat JM, Pieterman CR, van den Broek MF, TwiskJW, Hermus AR, Dekkers OM, et al. Natural course and survival of neuroendocrine tumors of thymus and lung in MEN1 patients. J Clin Endocrinol Metab. 2014 Sep;99(9):332533.

14 Goudet P, Murat A, Binquet C, Cardot-Bauters C, Costa A, Ruszniewski P, et al. Risk factors and causes of death in MEN1 disease. A GTE (Groupe d'Etude des Tumeurs Endocrines) cohort study among 758 patients. World J Surg. 2010 Feb;34(2):249-55

15 de Laat JM, Dekkers OM, Pieterman CR, Kluijfhout WP, Hermus AR, Pereira AM, et al. Long-Term Natural Course of Pituitary Tumors in Patients With MEN1: Results From the DutchMEN1 Study Group (DMSG). J Clin Endocrinol Metab. 2015 Sep;100(9): 3288-96.

16 Dean PG, van Heerden JA, Farley DR, Thompson GB, Grant CS, Harmsen WS, et al. Are patients with multiple endocrine neoplasia type I prone to premature death? World J Surg. 2000 Nov;24(11):1437-41.

17 Trump D, Farren B, Wooding C, Pang JT, Besser GM, Buchanan KD, et al. Clinical studies of multiple endocrine neoplasia type 1 (MEN1). QJM. 1996 Sep;89(9):653-69.

18 Albers MB, Manoharan J, Bollmann C, Chlosta MP, Holzer K, Bartsch DK. Results of Duodenopancreatic Reoperations in Multiple Endocrine Neoplasia Type 1. World J Surg. 2019 Feb; 43(2):552-8.

19 Albers MB, Manoharan J, Bartsch DK. Contemporary surgical management of the Zollinger-Ellison syndrome in multiple endocrine neoplasia type 1. Best Pract Res Clin Endocrinol Metab. 2019 Oct;33(5):101318.

20 Conemans EB, Nell S, Pieterman CR, de Herder WW, Dekkers OM, Hermus AR, et al. Prognostic factors for survival of MEN1 patients with duodenopancreatic tumors metastatic to the liver: results from the DMSG. Endocr Pract. 2017 Jun;23(6):641-8.

21 Romijn JA. Hyperprolactinemia and prolactinoma. Handb Clin Neurol. 2014;124:185-95.

22 Burgess JR, Giles N, Shepherd JJ. Malignant thymic carcinoid is not prevented by transcervical thymectomy in multiple endocrine neoplasia type 1. Clin Endocrinol (Oxf). 2001 Nov; 55(5):689-93.

23 Sakurai A, Imai T, Kikumori T, Horiuchi K, Okamoto T, Uchino S, et al.; MEN Consortium of Japan. Thymic neuroendocrine tumour in multiple endocrine neoplasia type 1: female patients are not rare exceptions. Clin Endocrinol (Oxf). 2013 Feb;78(2):248-54. 